\title{
The Web-Surf Task: A translational model of human decision-making
}

\author{
Samantha V. Abram ${ }^{1} \cdot$ Yannick-André Breton ${ }^{2} \cdot$ Brandy Schmidt $^{2} \cdot$ A. David Redish ${ }^{2}$. \\ Angus W. MacDonald, III ${ }^{1,3}$
}

Published online: 16 September 2015

(C) Psychonomic Society, Inc. 2015

\begin{abstract}
Animal models of decision-making are some of the most highly regarded psychological process models; however, there remains a disconnection between how these models are used for pre-clinical applications and the resulting treatment outcomes. This may be due to untested assumptions that different species recruit the same neural or psychological mechanisms. We propose a novel human foraging paradigm (WebSurf Task) that we translated from a rat foraging paradigm (Restaurant Row) to evaluate cross-species decision-making similarities. We examined behavioral parallels in human and non-human animals using the respective tasks. We also compared two variants of the human task, one using videos and the other using photos as rewards, by correlating revealed and stated preferences. We demonstrate similarities in choice behaviors and decision reaction times in human and rat subjects. Findings also indicate that videos yielded more reliable and valid results. The joint use of the Web-Surf Task and Restaurant Row is therefore a promising approach for functional translational research, aiming to bridge pre-clinical and clinical lines of research using analogous tasks.
\end{abstract}

Electronic supplementary material The online version of this article (doi:10.3758/s13415-015-0379-y) contains supplementary material, which is available to authorized users.

Angus W. MacDonald, III

angus@umn.edu

1 Department of Psychology, University of Minnesota, Twin Cities, 75 East River Parkway, Minneapolis, MN 55455, USA

2 Department of Neuroscience, University of Minnesota, 321 Church Street Southeast, Minneapolis, MN 55455, USA

3 Department of Psychiatry, University of Minnesota, 717 Delaware Street SE, Suite 516, Minneapolis, MN 55414, USA
Keywords Functional translation · Decision-making · Human · Rat · Impulsivity

\section{Background}

Animal models of impulsivity are regarded as being among the most well developed representations of human psychopathology (Kalivas, Peters, \& Knackstedt, 2006; Madden \& Bickel, 2010), and have been key contributors to our understanding of human psychopathologies, such as addiction (Madden \& Bickel, 2010; O'Brien \& Gardner, 2005). Nonetheless, there remains a gap between model validity and the efficacy of human treatments based on these animal models (Hall, De Serrano, Rodd, \& Tropepe, 2014; Kalivas et al., 2006). Prior research suggests this gap may stem from untested assumptions that humans and nonhuman animals recruit the same cognitive systems (Demeter, Sarter, \& Lustig, 2008). Coordinating human and non-human animal research to model the same behaviors is therefore critical to elucidating the behavioral and neurobiological mechanisms that underlie many psychopathologies (Belzung \& Lemoine, 2011; Potenza, 2009). However, this functional approach to translation requires parallel tasks that access similar functional constructs. Here, we present a novel experiential human foraging task translated from a rat food foraging paradigm (Steiner \& Redish, 2014). Instead of food, humans foraged for information through an internet-like interface, as a naturalistic analogue to the food rewards used with non-human animals (Pirolli, 2005). Our results suggest these tasks captured behavioral parallels in human and rat decision-making.

\section{The foraging model of decision-making}

New theories posit that many psychopathologies are fundamentally problems with decision-making. This notion implies 
that understanding the causes (and improving treatments) depends on understanding how those decision-making systems work and break down (Montague, Dolan, Friston, \& Dayan, 2012; Rangel, Camerer, \& Montague, 2008; Redish, Jensen, \& Johnson, 2008; Redish, 2013). Foraging models of decisionmaking provide a computational account of how humans and non-human animals allocate scarce resources (e.g., time) when searching for valuable resources like food, money, or drugs (Stephens, 2008). Sociological observations of drug-users suggest that users are seen as "foraging" for drugs in a "patchy" world of opportunities; for example, smokers looking for the cheapest cigarettes (Feighery, Schleicher, Boley Cruz, \& Unger, 2008; Grossman \& Chaloupka, 1998), gamblers looking for video poker machines (Schüll, 2012), or heroin addicts looking for narcotics (Hoffer, Bobashev, \& Morris, 2009). Thus, foraging paradigms may be a promising approach for examining the complex decision-making systems that underlie addiction or other psychopathological disorders.

Foraging models advance historical intertemporal choice models of decision-making, during which subjects make binary choices between rewards of different value that are available at disparate time delays (often referred to as "delaydiscounting" paradigms). Delay-discounting tasks have been widely used to assess impulsive decision-making among addicted human and non-human animals. However, multioption foraging paradigms may be more akin to real-world scenarios where humans are cognizant of other options or alternatives in the background when making a decision. Moreover, researchers have posited that stay/skip serial foraging choices may better characterize naturalistic decision-making (Stephens, 2008; Wikenheiser, Stephens, \& Redish, 2013). As a result, researchers are using foraging models at an increasing rate in both human and non-human animal studies (Hayden, Pearson, \& Platt, 2011; Kolling, Behrens, Mars, \& Rushworth, 2012; Shenhav, Straccia, Cohen, \& Botvinick, 2014; Steiner \& Redish, 2014; Wikenheiser et al., 2013). A logical next step is to develop a foraging model that translates across species; researchers could then use this foraging model to examine cross-species parallels in the maladaptive decision-making behaviors that support psychopathologies like addiction. This type of translation requires a bridging of research branches, which have typically produced methodologically divergent decision-making paradigms.

\section{Current challenges in functional translation}

Decision-making tasks for non-human animals are experiential, in that they typically entail a rat physically running through a maze or pressing a lever, waiting through realtime delays, and receiving primary reinforcers as reward, like food (Mazur, 1987; Papale, Stott, Powell, Regier, \& Redish, 2012). In contrast, efforts to produce comparable human experiential paradigms generally result in one of three approaches for reward stimuli: (i) secondary reinforcers like tallied points that may eventually convert to money (Kolling et al., 2012; Reynolds \& Schiffbauer, 2004; Shenhav et al., 2014), (ii) secondary reinforcers like coins that are dispensed during the task (Krishnan-sarin et al., 2007; Reynolds, 2006b; Voon et al., 2010), or (iii) primary rewards like juice or candy (Kool \& Botvinick, 2014; McClure, Ericson, Laibson, Loewenstein, \& Cohen, 2007). These methodological differences may impact the underlying reward systems evoked in humans. For example, in scenarios where (i) points/money are summated over the session or (ii) the subject randomly receives one or several of their choices at the end (the latter called real-reward measures; Reynolds, 2006a), each poor decision may be salient. This is because either: (i) each choice influences the ultimate gain or (ii) the subject does not know which final outcome the subject will receive (thus, making each decision important). On the contrary, in real-time measures, the subject consumes rewards at the end of each trial (Reynolds, 2006a). As a result, poor choices may be less salient as individual choices do not influence a post-session outcome. Given these distinctions, it is also possible that the same reward systems observed in rats are not evoked during human tasks that lack comparable real-time consummatory rewards.

Researchers have identified and addressed similar methodological gaps with respect to visuospatial paradigms. For example, human studies historically assessed spatial ability via paper and pencil tests, whereas non-human studies assessed spatial navigation via maze-learning tasks (Moffat, Hampson, \& Hatzipantelis, 1998). In response, many human studies adopted virtual reality radial mazes and Morris water tasks and successfully identified cross-species behavioral and neural parallels (Bohbot, Lerch, Thorndycraft, Iaria, \& Zijdenbos, 2007; Hamilton, Driscoll, \& Sutherland, 2002; Hamilton, Kodituwakku, Sutherland, \& Savage, 2003; Iaria, Petrides, Dagher, Pike, \& Bohbot, 2003). Despite the success of these virtual reality paradigms, these particular tasks did not address the issue of primary versus secondary reinforcement that may be pertinent to decision making (as subjects solely received monetary compensation at the study conclusion). Thus, experiential human foraging models with primary reinforcement are needed to fill this gap in external validity and provide a link to the animal decision-making literature.

\section{Primary reinforcement for humans}

Considering how humans interface with the world on a daily basis while seeking rewards or entertainment may improve insight into the processes underlying decision-making, which in turn may guide task development. Information Foraging Theory suggests that humans seek and acquire information using the Internet (Pirolli \& Card, 1999). More specifically, individuals perform ongoing cost-benefit analyses as they navigate through websites, making stay/skip foraging choices to remain on the 
current site or move on to the next (Pirolli, 2005). Humans also forage the Internet for rewarding stimuli, frequently presented in the form of video segments or images - each of which we can feasibly incorporate into an experimental paradigm. Such internet-found stimuli may even yield natural reinforcement that is comparable to drugs or food. Recent findings that Internetaddicted individuals exhibited functional and structural brain similarities to drug-addicted individuals bolster this claim (Ding et al., 2013; Kuss \& Griffiths, 2012; Weinstein \& Lejoyeux, 2015). The combined feasibility and primary reinforcement possible from using videos or photos as reward makes their use a compelling option for human task development.

\section{Study aims}

The current study advances available experiential tasks for humans by developing a foraging paradigm that (i) translates across species, (ii) includes primary reinforcement and realtime delays, and (iii) integrates natural human ethology into the design. We translated the proposed task directly from a novel stay/skip foraging paradigm, called "Restaurant Row" (Steiner \& Redish, 2014), during which a rat had a fixed amount of time to traverse a circular track and collect food rewards from four feeders. Each feeder (or "restaurant") provided a different flavor of food pellet after a random time delay (see Fig. 1A). We drew from Information Foraging Theory and the burgeoning Internet-addiction literature to develop the human variant of Restaurant Row, which we call the "Web-Surf Task" (see Fig. 1B). During this task, humans made a series of stay/skip decisions while traveling between galleries that contain primary rewards (videos or photos), which were presented after real-time delays. In this paper, we illustrate the external and face validity of the Web-Surf Task, as well as cross-species behavioral parallels using the Web-Surf Task and Restaurant Row.

\section{Methods and Materials}

\section{Web-Surf Task in humans}

\section{Sample demographics}

The total sample included 64 University of Minnesota undergraduates $(72 \%$ female, mean age $=20.5)$, who received extra credit toward a psychology course. The initial round of data collection included both the video $(\mathrm{N}=22)$ and photo versions $(\mathrm{N}=15)$; the second round of data collection included only the video version $(\mathrm{N}=27)$. This resulted in a total of 49 subjects who completed the video version. The University of Minnesota's Institutional Review Board approved the study, and all subjects provided written informed consent.

\section{Web-Surf Task design}

Subjects had $30 \mathrm{~min}$ to "surf" (or forage) through four galleries (see Fig. 2 for decision flow-diagram) presented using PsychoPy (Peirce, 2009). In the video variant, each gallery presented a video clip from one of four categories (kittens, dance, bike-accident, landscapes) as a reward. In the photo variant, each gallery presented an image from one of four categories (kittens, desserts, female faces, or landscapes); we note that we transformed the still images using the Ken Burns panning and zooming effect to parallel the video variant. We selected these particular categories in consideration of future functional neuroimaging data collection and neural decoding analyses. More specifically, we expect these categories will map onto separable neural substrates, as prior evidence indicates unique correlates for faces, bodies, animals, natural scenes, tools/objects, and animals (Bradley, Katherine, Dylan, Daphna, \& Nathaniel, 2015; Haxby et al., 2001, 1999; Peelen \& Downing, 2007; Walther, Caddigan, Fei-Fei, \& Beck,

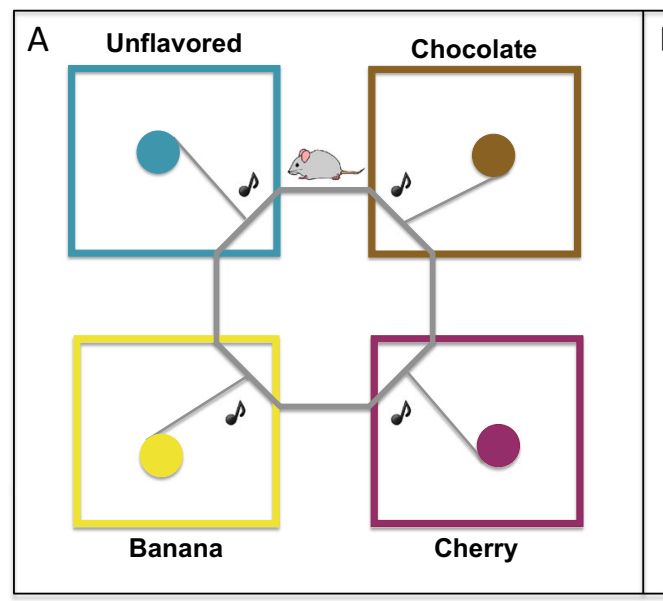

Fig. 1 (A) Schematic representation of Restaurant Row. Rats had 60 min to cycle around a circular track and collect food rewards from four feeders ("restaurants"); feeders provided different flavors of pellets after variable

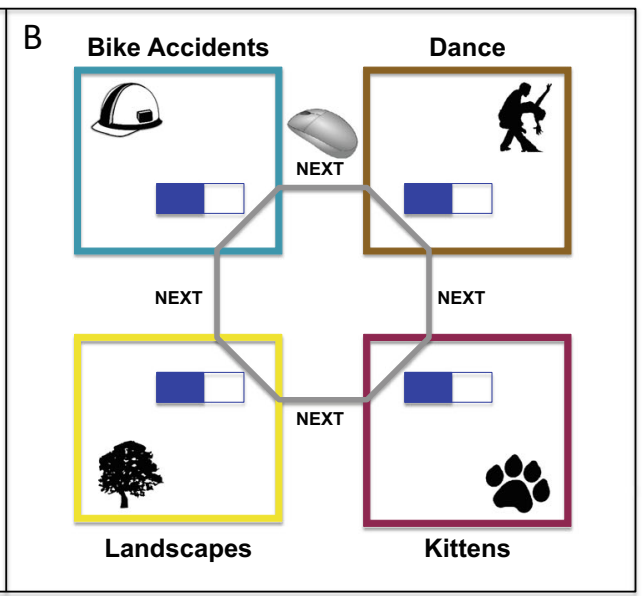

delay times. (B) Schematic representation of the Web-Surf Task. Humans had $30 \mathrm{~min}$ to cycle through four video or photo galleries; video or photo rewards were represented after variable delay times 


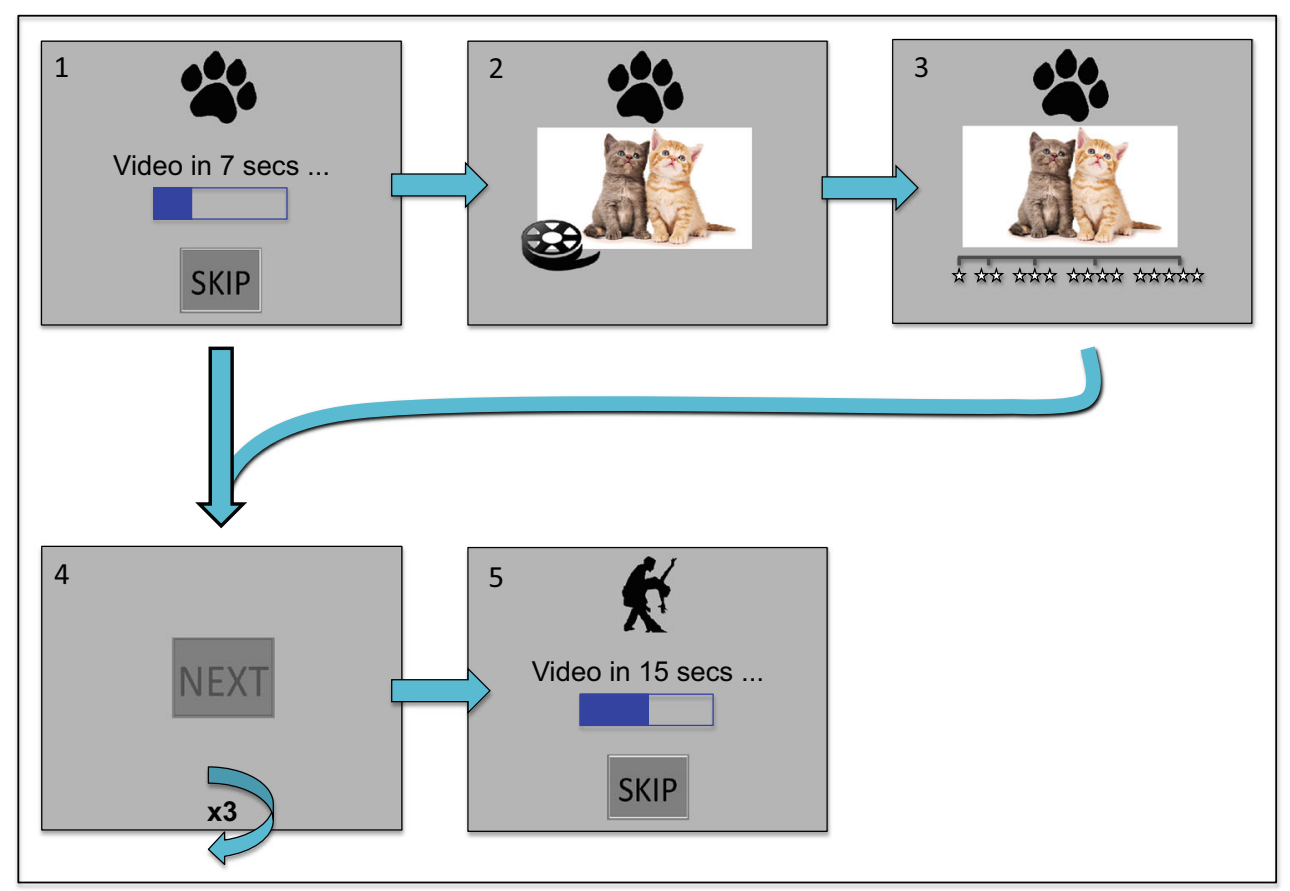

Fig. 2 Flow diagram of the Web-Surf Task to illustrate stay/skip decisions. Subject receives an offer (1). If the subject decides to stay: the subject views the video or photo stimulus for four s (2), rates the stimulus at the end of four s (3), proceeds through the "NEXT" transition phase

2009). For additional details on the anticipated decoding analyses see Steiner and Redish (2014).

We laid out the task as follows: Upon arrival at a gallery, the subject was informed of the random delay time before video presentation. Delay time was displayed using text and a progress bar similar to those located on an Internet webpage. The subject was given the option to stay and wait for the current reward or skip and continue on to the next gallery. If the subject decided to stay, the subject viewed the stimulus for four seconds and then rated it using a five-star rating system (one star = extremely dislike, five stars = extremely like). If the subject decided to skip, the subject pushed the "SKIP" button located at the bottom of the screen. After leaving the gallery, the subject "surfed" to the next gallery that presented a new offer (i.e., new video or photo after a new random delay). The subject then completed a series of "NEXT" screens when traveling between galleries, regardless of the decision to stay or skip; this entailed finding and clicking three or five "NEXT" buttons that were randomly positioned on the screen. We intended the "NEXT" to serve as an analogue to the rats physically running a track to travel between feeders. We designed the buttons to blend into the background to increase the cost for locating them around the screen. Twenty-two subjects completed the task with five "NEXT" screens between galleries; 27 subjects completed the task with three "NEXT" screens between galleries.

As preliminary training, subjects completed two forced practice trials, during which we instructed them to push the "SKIP" button for trial one and to stay and wait for trial two. We created
(4), and then receives the next offer (5). If the subject decides to skip: the subject moves directly from the initial offer (1) to the "NEXT" transition phase (4), before receiving the next offer (5)

this structure to illustrate the two choice options as well as the transition between the galleries. Subjects then completed eight practice trials where they could decide whether to stay or skip.

\section{Restaurant Row in rats}

\section{Sample characteristics}

We used eight adult Brown-Norway rats in this experiment. Our methods were consistent with Steiner and Redish (2014), as we aimed to replicate behavioral findings in a new sample. Our study protocol complied with the National Institute of Health guidelines for animal care, and the Institutional Animal Care and Use Committee at the University of Minnesota approved the protocol.

\section{Restaurant Row design}

Restaurant Row consisted of a circular track with four spokes leading off to food-reward sites (restaurants) as illustrated in Fig. 1A. Each food-reward site provided a different flavor of food (cherry, chocolate, banana, and unflavored/plain sugar). The rat proceeded around the circle encountering each offer serially. When the rat entered the offer zone, a tone sounded, with pitch indicating the delay (1-30s). The tone counted down once per second (change $=250 \mathrm{~Hz}$ ) until it reached the base tone (one $\mathrm{kHz}$ ), at which time the two pellets of the flavor for that restaurant were delivered. If the rat left the offer 
zone before the delay finished counting down, the tone stopped, the offer was rescinded, and the rat had to proceed to the next restaurant to get food. Because zones were only triggered in a clockwise serial manner, rodents quickly learned to run in one direction. Essentially, the animal made a series of stay/skip decisions, such as - Is it worth waiting $25 \mathrm{~s}$ for two banana-flavored food pellets? In this task, we gave rodents $60 \mathrm{~min}$ to collect food for the day. The 60 -min time limit means that the encounters were not independent of each other - time spent waiting at one restaurant was time that could not be spent waiting at another. This means that an animal using an economically intelligent strategy should have waited longer for more preferred flavors. Rats' preferences were "revealed" by an increased willingness to wait out a longer delay for a favored flavor of pellet. Each rat completed nine or ten sessions in total.

We trained rats in four phases. In the first phase (5-7 days, twice daily), rats completed 30-min sessions of habituation. The delay at every feeder was one $\mathrm{s}$ and the reward was two pellets. During this phase the rat became accustomed to the task, whereby it learned the correct direction of travel and the flavor available at each feeder site. The rat moved on to the next training phase after it reliably ran clockwise around the loop. In the second phase (four days, twice daily), the 30-min sessions had increasingly longer delays. The delays began with a range of 1-2 s, then 1-3 s, and continued to increase by one s each day until the rat achieved a maximum of five $\mathrm{s}$ (this trained the rat to wait). In the third phase (10 days, twice daily), the rat completed 30-min sessions with the full delay set (1-30 s). In the fourth phase (5-10 days, once daily), the rat completed 60-min sessions with the full delay set (1-30 s). By the end of this final training phase, the rats typically showed delay thresholds (by visual inspection), skipped high tones, and left the feeder site after reward receipt. From this evidence, we concluded that the rats understood the task and commenced the experimental testing portion.

\section{Task-derived decision metrics}

To examine behavioral parallels across species we used three decision metrics: (i) revealed preferences, (ii) stated preferences, and (iii) decision consistency. We calculated revealed preferences for each category via a logistic fit function for human and non-human animals (see Fig. 3). These values reflect the delay time (or delay threshold) at which a subject reliably began to skip offers for the respective category. In other words, the inflection point equates to the delay threshold at which a subject had a $50 \%$ probability of staying (or skipping). We computed inflection points according to the following equation (one per category, per subject):

$\log \left(\frac{p}{1-p}\right)=\beta_{0}+\beta_{1} x$ where $p$ is the desired probability ( $50 \%$ in this case), $\beta_{0}$ is the intercept, $\beta_{1}$ is the slope, and $x$ is the delay threshold. Notice that the function on the left of equation (1) is the logit of $p$, and the function on the right of equation (1) represents a linear regression model with a single predictor. We then rearranged the equation to solve for $x$ :

$x=\frac{\log \left(\frac{p}{1-p}\right)-\beta_{0}}{\beta_{1}}$

We considered favored galleries to be those where a subject consistently waited longer for the reward (equating to a higher delay threshold). We acquired stated preferences for human subjects only, which included average ratings for each category, as well as post-test category rankings, one to four.

We also measured decision consistency in both species, which indicated the extent to which subjects cohered to categoryspecific strategies (i.e., stayed for trials below threshold, and skipped trials above threshold). Given the economically normative assumption that subjects had a subjective valuation of a particular category and a fixed time constraint, subjects should have stayed when the subjective valuation of a category (reward) was larger than the offered delay (cost). Subjects that deviated from this economically normative model made "economic errors" in that they sacrificed time that could be spent in a preferred location (e.g., time spent waiting for a bike-accident video was time that could not be spent waiting for a [potentially preferred] kitten video). To derive the decision consistency metric we computed the proportion of error trials for each subject (number of error trials divided by the total number of trials).

\section{Analyses}

Our first set of analyses assessed behavioral cross-species parallels using the respective tasks. First, we identified evidence of revealed preferences in both species. Next, we evaluated withinsubject consistency. For human subjects, we examined the correspondence between a human subject's revealed and stated preferences; this analysis also provided a measure of the WebSurf Task's external validity. For rats, we evaluated the consistency of the rats' revealed preferences (i.e., delay thresholds) across sessions because we could not ask a rat to explicitly state its preference. Third, we investigated whether humans and nonhuman animals exhibited similarities in decision consistency. Fourth, we analyzed decision times to determine whether subjects made quick decisions or waited for cues; we used this measurement to evaluate the face validity of each task. Finally, we examined within-session dynamics to determine whether humans and rats behaved similarly as the session proceeded.

In the second set of analyses, we compared the two variants of the Web-Surf Task (video vs. photo stimuli). We conducted these analyses to determine which type of stimuli provided the most reliable and valid results, while also considering category 


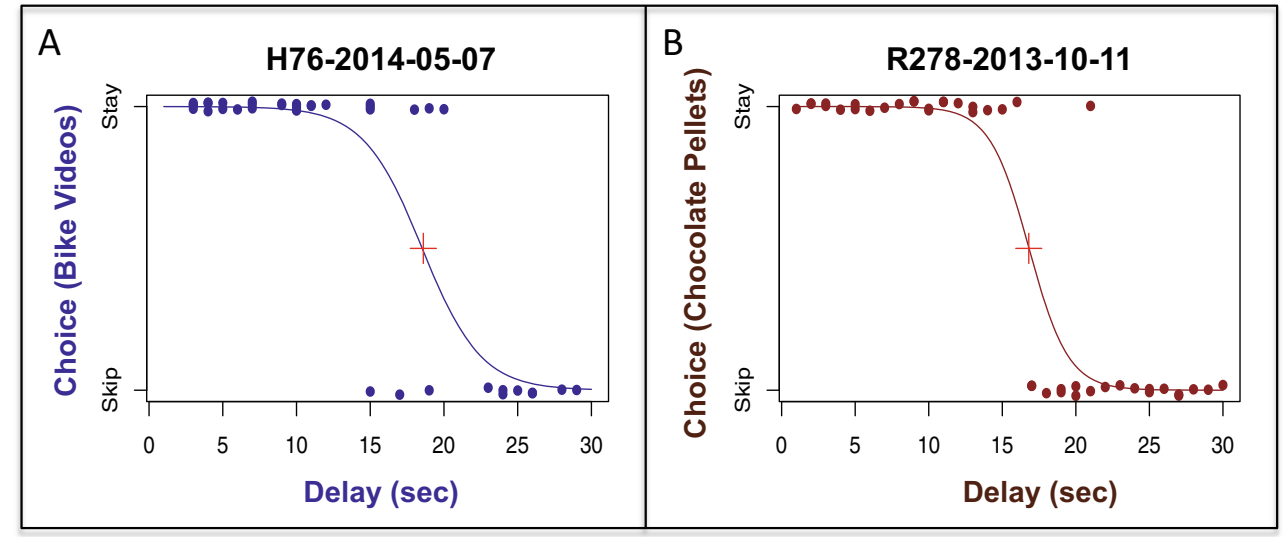

Fig. 3 Examples of subject level plots for a single category for the WebSurf Task (A) and Restaurant Row (B), which show revealed preferences. The red plus sign indicates a subject's inflection point, or the delay

homogeneity across stimuli types (rats always received the same reward at a given feeder). We also assessed within-session dynamics and gender differences across the two task variants.

\section{Results}

\section{Humans versus rats: Revealed preferences}

First, we determined whether human and non-human animal subjects showed evidence of revealed preferences. Figure 3 illustrates a side-by-side comparison for a single human and single rat session, where each curve depicts choices for a particular category. In both species, we visually (and statistically) identified a clear inflection point at which the subject had a 50 $\%$ chance of staying or skipping on to the next offer. The distributions in Fig. 3 are typical of those we observed from other human and non-human animal subjects (see Steiner \& Redish, 2014, for additional rodent examples).

\section{Humans versus rats: Evaluating within-subject consistency}

Next, we evaluated the extent to which both species displayed consistent within-subject preferences. For the human sample ( $\mathrm{N}=49$ ), we computed two correlations per subject: (i) the correlation between delay thresholds and average category ratings (four values for each) and (ii) the correlation between delay thresholds and post-test category rankings (four values for each). We found that delay thresholds corresponded with average category ratings, with $69 \%$ of correlations above 0.50 . Similarly, $73 \%$ of correlations between delay thresholds and post-test category rankings were above 0.50 . We did not detect significant differences between subjects who completed the three versus five NEXT versions for rating $\left(\mathrm{t}_{47}=0.51, p=\right.$ $0.61)$ or ranking $\left(\mathrm{t}_{38}=0.08, p=0.94\right)$ validity correlations. threshold at which a subject reliably began to skip offers for a given category (calculated using a logistic fit function). These are typical distributions (see Steiner \& Redish, 2014)

To determine within-subject consistency for rats, we evaluated delay thresholds across sessions using a repeatedmeasures analysis of variance (ANOVA). We constructed a model that included delay thresholds as the dependent variable, and zone (i.e., feeder site) and session number as the predictor variables. As shown in Table 1, we observed a significant main effect for zone $\left(\mathrm{F}_{3,231}=4.58, p=0.004\right)$ but not session number $\left(\mathrm{F}_{70,231}=0.59, p=0.99\right)$, thus indicating that rats had detectable and stable flavor preferences. These results are consistent with across-session rat performance in Steiner and Redish (2014).

\section{Humans versus rats: Decision consistency}

Third, we examined parallels in decision consistency for humans and non-human animals (see Fig. 4). In particular, we found that rat subjects exhibited greater decision instability (mean $=0.12$, median $=0.12)$, compared to human subjects (mean $=0.07$, median $=0.08$ ). Several human subjects also had no error trials, which was not the case for rats. We were also interested in the spread of this decision consistency metric within each species, as we hope to capture a comparable range of threshold error variance using the respective tasks. To this end, we used an F-test to investigate differences in decision consistency variance between species and found no significant differences $\left(\mathrm{F}_{49,77}=1.15, p=0.60\right)$. Thus, although humans had less mean decision instability, the spread of this metric was consistent in humans and rats.

Table 1 Across-session threshold consistency (rat subjects, $\mathrm{N}=8$ )

\begin{tabular}{lllcll}
\hline Source & df & Sum sq & Mean sq & F-value & $p$-value \\
\hline Zone & 3 & 523 & 174.27 & 4.58 & $\mathbf{0 . 0 0 4}$ \\
Session Number & 70 & 1,581 & 22.59 & 0.59 & 0.99 \\
Residuals & 231 & 8,788 & 38.04 & & \\
\hline
\end{tabular}




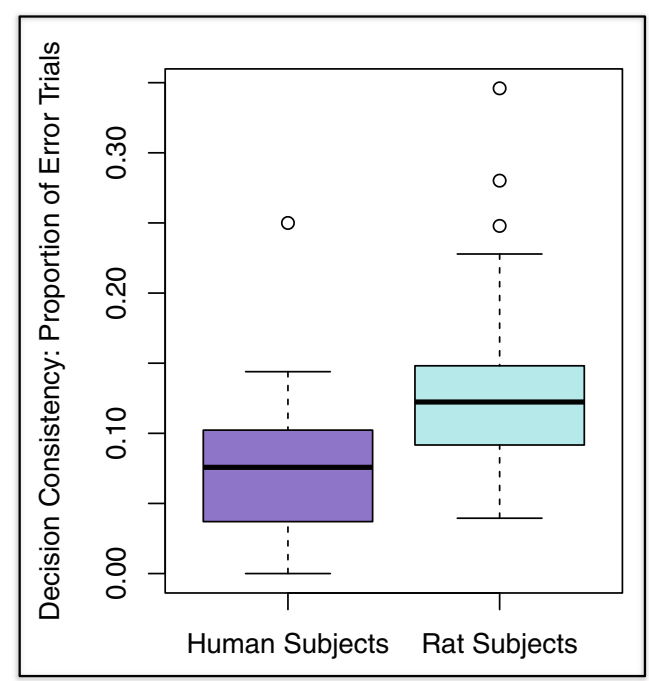

Fig. 4 Distributions representing decision consistency for the Web-Surf Task (right) and Restaurant Row (left). Specifically, this metric reflects the proportion of trials for which a subject deviated from his or her strategy (skipped an offer below his delay threshold for the respective category or stayed for an offer above his threshold). Here, the upper and lower bars indicate the range of scores, the shaded area depicts the interquartile range, and the dark horizontal band reflects the median decision consistency score. Circles outside the boxes show outliers

\section{Humans versus rats: Decision times}

Fourth, we evaluated the association between choice reaction time and delay to determine whether delay times influenced the speed at which subjects made a decision. This analysis included a subset of human video subjects $(\mathrm{N}=27)$ for whom the task recorded skip reactions times. The plots in Fig. 5 illustrate the relation between choice reaction time and delay for all human and rat subjects separately. Stay trials are represented as the full delay time (points along diagonal) and mean times for skip trials are represented as the points parallel to the $\mathrm{x}$-axis. The blue shaded bands indicate skip time standard deviations around each possible delay time. Humans and rats made decisions shortly after arrival at a gallery, with decisions made within three s or less for the majority of human trials and within five $\mathrm{s}$ or less for the majority of rat trials. In other words, when presented with a less valuable offer, subjects did not hesitate to skip and travel towards other potential offers. Instead, both species efficiently decided whether an offer was preferable or not. This supports the face validity of each task, where neither species waited for a specific cue to decide but made a quick choice and remained engaged in the task. For example, the rats did not appear to wait for a specific tone before deciding to leave.

\section{Humans versus rats: Within-session dynamics}

As a final cross-species comparison, we assessed whether human and rat behaviors showed comparable fluctuations throughout the session. To this end, we used repeated measures ANOVAs with choice as the dependent variable, and category/zone and trial number as the predictor variables. We were particularly interested in the trial number term as an indicator of reward satiation, where a significant effect would suggest a change in stay/skip tendencies over the session. Table 2 reveals a significant main effect for category $\left(\mathrm{F}_{3}\right.$, $\left.{ }_{5587}=110.78, p<0.001\right)$ but not trial number $\left(\mathrm{F}_{1,5587}=2.03, p\right.$ $=0.16$ ) for human subjects. Table 3 reveals comparable findings for rat subjects, including a significant main effect for zone $\left(\mathrm{F}_{3,13856}=18.69, p<0.001\right)$ but not trial number $\left(\mathrm{F}_{1}\right.$, $13856=1.28, p=0.25)$. This suggests that, regardless of species, subjects exhibited differential choice patterns across reward sites but not trial number. In other words, neither species appeared to satiate during the session.

We also assessed the amount of time spent in the reward zone following reward consumption as a function of trial number. In human subjects this equated to the time after viewing a stimulus but before providing a rating (as the subject could not advance to the next gallery before rating the video or photo).
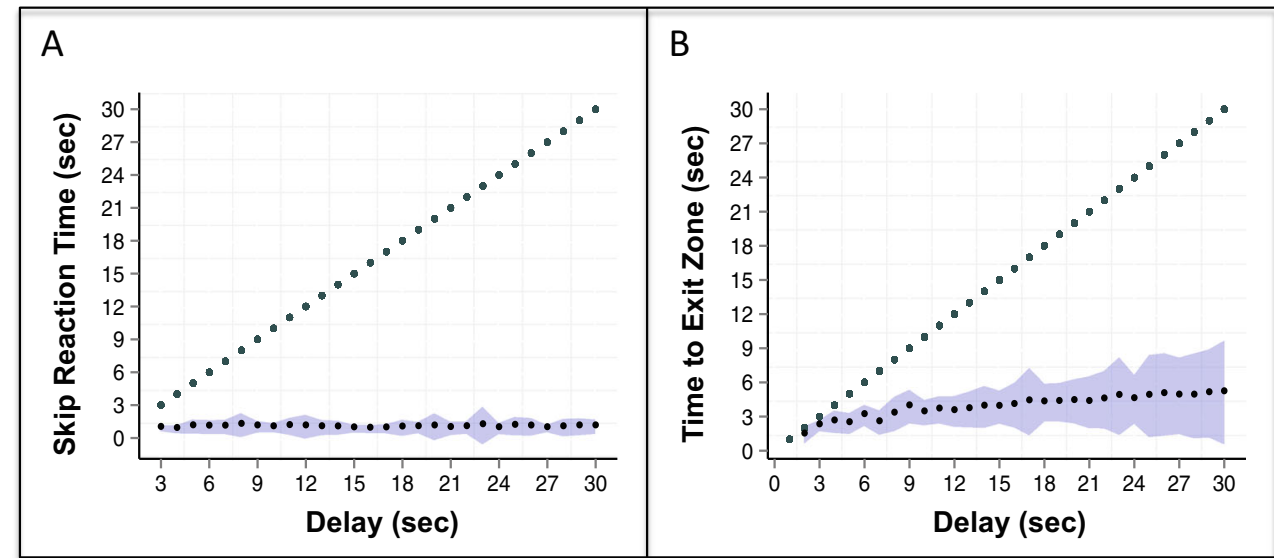

Fig. 5 Choice reaction times for the Web-Surf Task (A) and Restaurant Row (B). Stay trials shown as the full delay time (points along the diagonal) and mean times for skip trials shown as the points parallel to the $\mathrm{x}$ - axis. Blue shaded bands indicate skip time standard deviations. Decisions were generally made quickly for humans and rats 
Table 2 Choice as a function of category and trial number (video subjects, $\mathrm{N}=49$ )

\begin{tabular}{lllcll}
\hline Source & df & Sum sq & Mean sq & F-value & $p$-value \\
\hline Category & 3 & 65.70 & 21.91 & 110.78 & $<\mathbf{0 . 0 0 1}$ \\
Trial Number & 1 & 0.40 & 0.40 & 2.03 & 0.16 \\
Residuals & 5,587 & $1,104.90$ & 0.20 & & \\
\hline
\end{tabular}

In rat subjects this equated to the time after eating but before running to the next zone. A t-test indicated that rats spent significantly more time lingering in the reward zone than humans, $\mathrm{t}_{12207}=46.23, p<0.001$ (see Figs. 6A and B).

\section{Videos versus photos: Selecting optimal stimuli}

To compare the task variants, we first computed correlations between revealed and stated preferences for the photo $(\mathrm{N}=$ $15)$ and a subset of the video subject $(\mathrm{N}=22)$ whose data we collected during the same period. For average category ratings, $68 \%$ of video subjects had correlations of 0.50 or above versus $40 \%$ of photo subjects. As depicted in Fig. 7A, video subject correlations for average category ratings ranged from -0.31 to 1.00 , with a mean of 0.62 and a median of 0.82 . The photo subject correlations spanned a comparable range from -0.76 to 1.00 . However, the mean and median correlations for the photo subjects were lower, with values of 0.27 and 0.35 , respectively. A t-test revealed a significant difference between the groups $\left(\mathrm{t}_{35}=-2.10, p=0.04\right)$.

A similar pattern emerged for the post-test category rankings, where $72 \%$ of video subjects had correlations 0.50 or above versus $64 \%$ of photo subjects (see Fig. 7B). Video subject correlations for category rankings ranged -0.40 to 0.99 , with a mean of 0.56 and a median of 0.85 . The photo subject correlations covered an even larger range, with bounds of -0.73 and 0.96 . As a result, the mean correlation for these subjects was 0.41 and the median 0.55 . Although video subjects generally had higher validity correlations, this difference was not significant $\left(\mathrm{t}_{27}=-0.73, p=0.47\right)$; eight subjects did not have ranking data, hence the reduced degrees of freedom.

We also calculated decision consistency metrics for photo subjects and the same subset of 22 video subjects. As shown in Fig. 8, video and photo subjects did not exhibit mean differences in their proportion of error trials $\left(t_{35}=-0.65, p=\right.$

Table 3 Choice as a function of zone and trial number (rat subjects, $\mathrm{N}=8$ )

\begin{tabular}{llllll}
\hline Source & df & Sum sq & Mean sq & F-value & $p$-value \\
\hline Zone & 3 & 12.20 & 4.07 & 18.69 & $<\mathbf{0 . 0 0 1}$ \\
Trial Number & 1 & 0.30 & 0.28 & 1.28 & 0.25 \\
Residuals & 13,856 & $3,015.80$ & 0.22 & & \\
\hline
\end{tabular}

0.52). Similar to the human and rat comparison, we also assessed for differences in decision consistency variance. Here we did find a significant difference $\left(\mathrm{F}_{21,14}=0.19, p<\right.$ $0.001)$, whereby video subjects exhibited a more homogenous pattern of decision instability.

\section{Videos versus photos: Within-session dynamics}

Next, we assessed within-session choice behaviors for the two task variants using repeated measures ANOVAs. This entailed two models with choice as the dependent variable, and category and trial number as the predictor variables (i.e., separate models for the initial 22 video subjects and the 15 photo subjects). Table 4 reveals a significant main effect for category $\left(\mathrm{F}_{3,2382}\right.$ $=64.35, p<0.001)$ but not trial number $\left(\mathrm{F}_{1,2382}=0.12, p=\right.$ 0.73 ) for video subjects. Table 5 reveals similar findings for photo subjects, including a significant main effect for zone $\left(\mathrm{F}_{3,1241}=8.85, p<0.001\right)$ but not trial number $\left(\mathrm{F}_{1,1241}=\right.$ $1.72, p=0.19)$. Thus, subjects showed significant choice differences as a function of category but not trial duration.

We also investigated whether video and photo subjects differed in the amount of time spent in the reward zone following consumption (after viewing a stimulus but before rating it). As shown in Figs. 9A and B, we observed similar patterns using the two task variants $\left(\mathrm{t}_{2272}=0.85, p=0.39\right)$, as human subjects generally rated the videos and exited the reward zone in five s or less (with comparable variation extending into the 5to 18 -s range).

\section{Videos versus photos: Gender differences}

Lastly, we built linear mixed models to assess gender differences in category preference; this approach uses restricted maximum likelihood to obtain parameter estimates and can thus accommodate unbalanced designs (i.e., missing data). We constructed two models per task variant (four models total) that included either delay thresholds or average category ratings as the dependent variable, and gender, category, and a gender $\mathrm{x}$ category interaction term as the predictor variables. We were particularly interested in the interaction term as an indicator of preference differences across gender. We observed non-significant interactions in all models (see Supplemental Tables $1 \mathrm{~A}-2 \mathrm{~B})$. However, a trend-level gender $\times$ category interaction for average category ratings in the video subjects $(\mathrm{N}=22)$ and a subsequent power analysis encouraged us to re-assess for significant interactions using the complete video sample $(\mathrm{N}=49)$. Here, we found significant gender $\times$ category interactions for delay thresholds $\left(\mathrm{F}_{3,141}=2.77, p=\right.$ $0.04)$ and average category ratings $\left(\mathrm{F}_{3,138}=6.12, p<0.001\right.$; see Supplemental Tables $3 \mathrm{~A}$ and $3 \mathrm{~B}$ ). Follow-up tests revealed that gender differences were most prominent for the bike-accident and landscape categories. We refer readers to the Supplementary Materials for further details. 


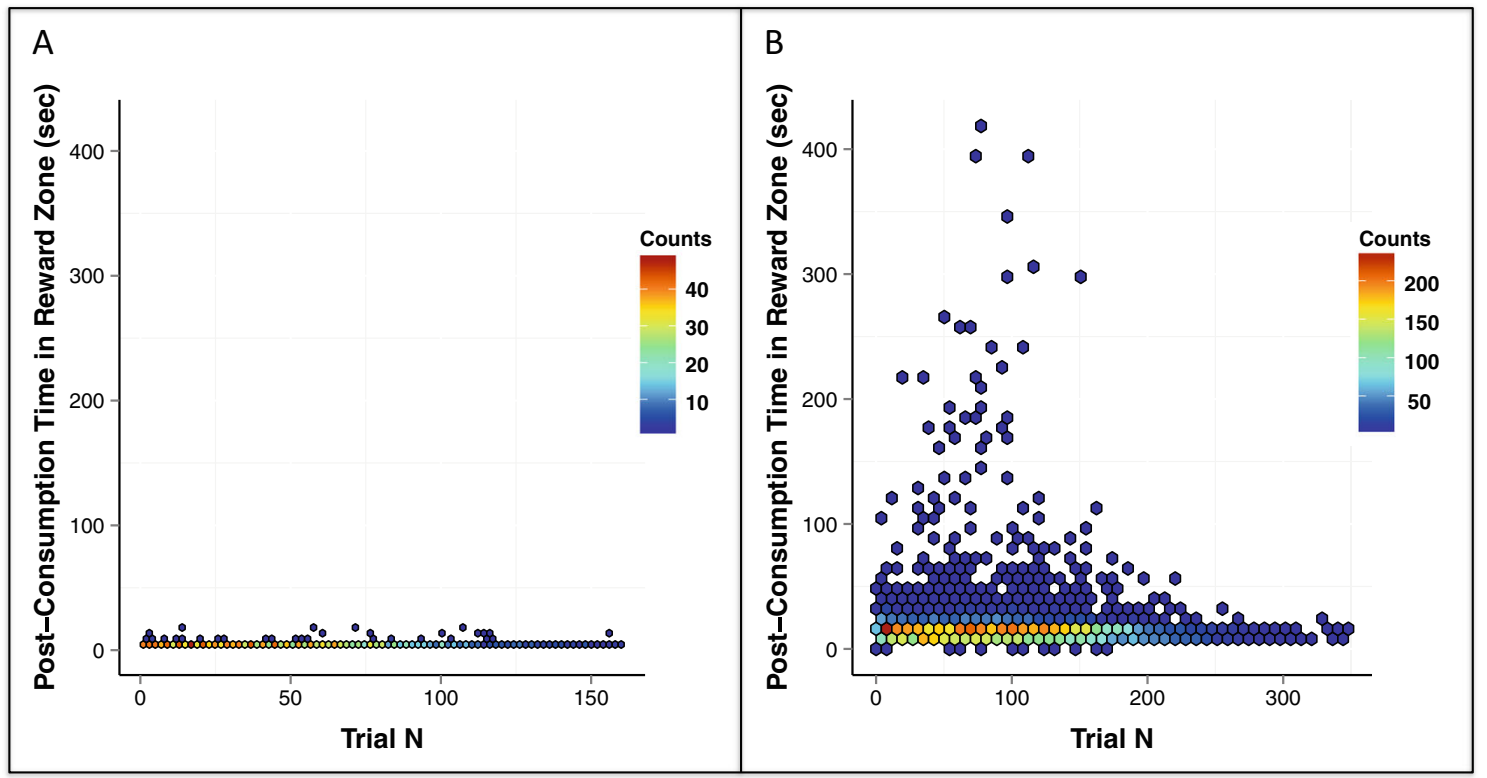

Fig. 6 Time spent in the reward zone after consumption against trial number (N) for the Web-Surf Task (A) and Restaurant Row (B). In human subjects this equated to the time after viewing a stimulus (video or photo)

\section{Discussion}

The current study proposes a novel experiential foraging paradigm for humans called the Web-Surf Task. We designed this paradigm to assess similarities in decision-making systems in humans and rats. The Web-Surf Task involves individuals making a series of stay/skip foraging decisions as they cycle through four galleries. This task builds on available decision-making paradigms in several ways: (i) its experiential design includes primary reinforcement and real-time delays, (ii) it entails serial but before providing a rating. In rat subjects this equated to the time after eating but before running to the next zone. The counts reflect the number of samples included in a given cell

stay/skip offers and is therefore more akin to real-world choices, and (iii) it was designed as a direct analogue to a rat foraging task. This last point is particularly salient in the context of psychopathology research, where translational models are critical for developing successful treatments. Our preliminary findings demonstrate both the external and face validity of the Web-Surf Task, as well as cross-species behavioral parallels using the analogous tasks. Therefore, the complementary use of the Web-Surf or Restaurant Row Tasks could be a step forward for bridging pre-clinical and clinical lines of research.

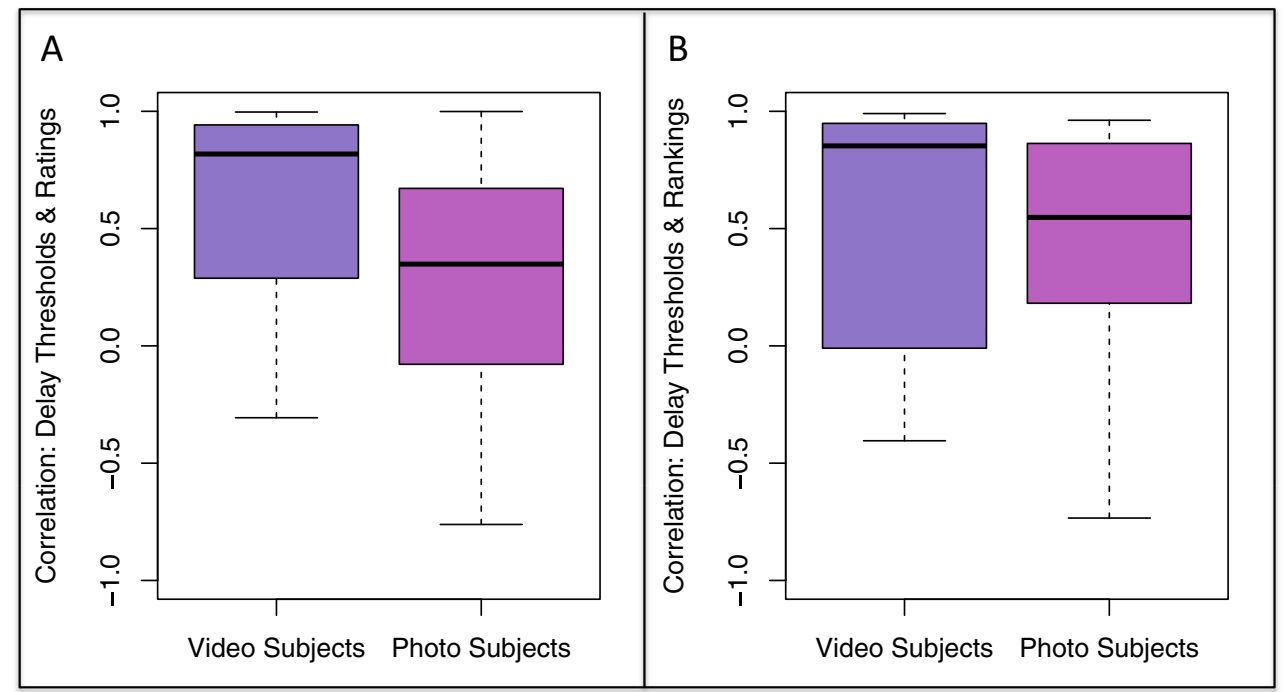

Fig. 7 Correspondence between revealed and stated preferences for both stimuli types. (A) Correlations between delay thresholds (revealed preferences) and average category ratings (stated preferences) for video and photos subjects. (B) Correlations between delay thresholds and post- test category rankings (stated preferences). The upper and lower bars represent the range of correlations. The shaded area represents the interquartile rang, and the dark horizontal band within the shaded area indicates the median correlation 


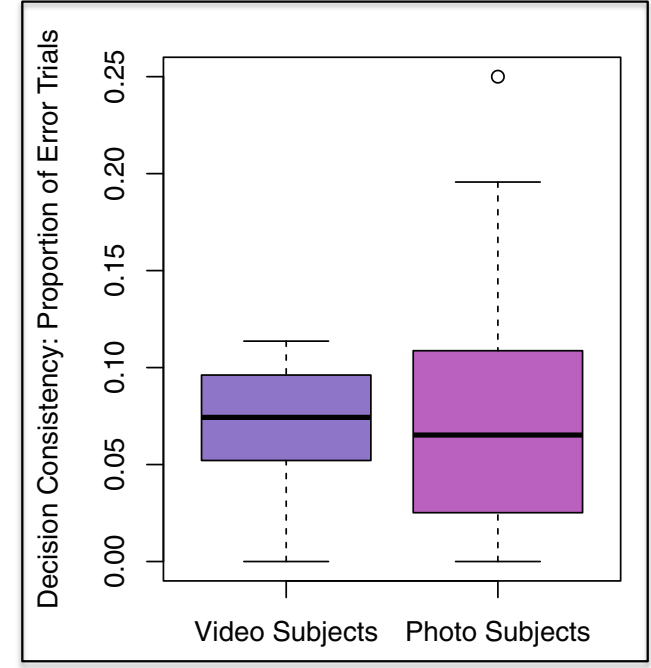

Fig. 8 Comparison of video versus photo subjects on measure of decision consistency. The upper and lower bars indicate the range of scores, the shaded area depicts the interquartile range, and the dark horizontal band reflects the median decision consistency score. Circles outside the boxes show outliers

We first examined cross-species parallels using data from both the Web-Surf Task and Restaurant Row. Our results showed that each task captured individual differences in preference as evidenced by delay thresholds, as well as within-subject consistency in humans and non-human animals. We also found evidence that both species actively made decisions as they traversed through their respective tasks, where each offer (combination of delay length with specific gallery or flavor) represented a certain value that fit within a given subjects' strategic framework. Moreover, we detected cross-species parallels in reward satiation rates, as tendencies to stay versus skip remained relatively stable throughout the session. We did observe crossspecies divergences with respect to decision consistency, where rat subjects exhibited more deviations, on average, from the ideal strategy. However, the spread of decision instability was similar using the analogous tasks. The tasks also diverged according to post-consumption reward time. In particular, we found that rat subjects spent more time lingering in the reward zone partaking in leisure activities such as grooming.

We then compared two variants of the Web-Surf Task: one that included video stimuli as reward and a second that included photo stimuli. Our primary intention was to empirically assess which type of internet-available reward stimuli yielded more

Table 4 Choice as a function of category and trial number (video subjects, $\mathrm{N}=22$ )

\begin{tabular}{llrcll}
\hline Source & df & Sum sq & Mean sq & F-value & $p$-value \\
\hline Category & 3 & 37.90 & 12.63 & 64.35 & $<\mathbf{0 . 0 0 1}$ \\
Trial Number & 1 & 0.00 & 0.02 & 0.12 & 0.73 \\
Residuals & 2,382 & 467.40 & 0.20 & & \\
\hline
\end{tabular}

Table 5 Choice as a function of category and trial number (photo subjects, $\mathrm{N}=15$ )

\begin{tabular}{llrlll}
\hline Source & df & Sum sq & Mean sq & F-Value & $p$-value \\
\hline Category & 3 & 4.84 & 1.61 & 8.85 & $<\mathbf{0 . 0 0 1}$ \\
Trial Number & 1 & 0.31 & 0.31 & 1.72 & 0.19 \\
Residuals & 1,241 & 226.24 & 0.18 & & \\
\hline
\end{tabular}

reliable and valid results. We found that subjects who completed the video version showed greater correspondence between revealed and stated preferences. We also observed a tighter range of decision stability in the video subjects. Hence, although photo categories may appear more homogenous, the data suggest that video rewards yielded more reliable results. These discrepancies may reflect the notion that videos are inherently more rewarding to humans. Comparable findings have been reported in macaques, where animated movies had considerably more reward value than static pictures (Blatter \& Schultz, 2006). We also compared within-session dynamics across the two task variants, which indicated similar stay proportions and postreward consumption times. As a last step, we explored gender differences in category preference. These results suggested that males tended to wait longer for landscape and bike-accident videos, and also rated these categories more highly (although the bike-accident ratings did not attain significance). We were unable to detect gender differences using the photo version, further suggesting an increased sensitivity in the video version.

Previous studies have demonstrated the utility of multioption foraging models for investigating natural foraging behaviors in human and non-human animals; however, ours is the first to compare these processes across species. The WebSurf Task provides a novel combination of primary reinforcement, real-time delays, and serial stay/skip foraging choices that parallels Restaurant Row.

\section{Future directions}

Despite the promising overlap of the described tasks, functional translation is a dynamic and evolving process that benefits from ongoing modifications at both the pre-clinical and clinical ends. We therefore suggest several avenues to further reduce cross-species divergences. First, future studies could assess whether decision-making parameters derived from the Web-Surf Task are stable via repeated sessions. This approach would not only foster design parallels with non-human animal studies (which typically entail multiple sessions) but also elucidate whether the Web-Surf Task captures state and/or traitlike effects. Some researchers argue that experiential decisionmaking tasks better capture acute state changes (e.g., drug effects), whereas questionnaire-based tasks may tap into stable, trait-like impulsivity (Reynolds, 2006a). Nonetheless, empirical research is needed to assess if the Web-Surf Task, 


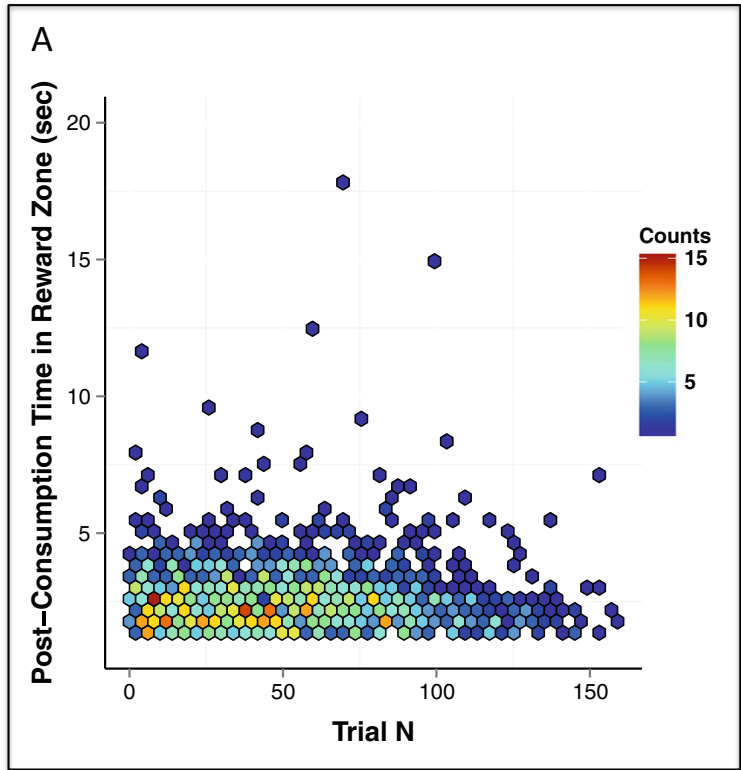

Fig. 9 Time spent in the reward zone after consumption against trial number $(\mathrm{N})$ for the Web-Surf Task, where $(\mathbf{A})$ is the initial 22 video subjects and (B) is the photo subjects. This equated to the time after

which we consider an experiential measure, can measure state-level fluctuations in a similar fashion to Restaurant Row. Second, researchers could investigate the extent to which various experimental design manipulations (e.g., increasing delay lengths, adjusting distance/effort to travel between feeders or galleries) similarly influence human and rodent behavior. Third, future endeavors could modify the stimuli sets to address specific psychopathology questions (e.g., food pictures or videos for obesity hypotheses, drug paraphernalia for addiction hypotheses, etc.). In effect, such stimuli would serve as a combination of primary and conditioned reinforcement. Researchers might then investigate whether satiation rates for these stimuli differ from other primary rewards. Fourth, researchers may examine relations between error trials and psychopathology, particularly the influence errors might have on trial-by-trial behavior. For example, a subject might encounter an unfavorable scenario where one skips an offer below threshold (where they should have stayed) only to encounter a less favorable offer on the next trial (termed "regret" when seen in rats by Steiner \& Redish, 2014). The manner in which a subject uses this experience to guide subsequent decisions may reflect pathological processes. For instance, addicted individuals may continue to deviate from strategy despite negative feelings or repercussions.

Another pertinent avenue for future endeavors is to explore the underlying neural systems evoked during the analogous tasks. Although we restricted the current study to behavioral methods, prior investigations have identified the rodent neural systems recruited during Restaurant Row (Breton, Schmidt, \& Redish, 2014; Schmidt, Breton, \& Redish, 2014; Steiner \& Redish, 2014). Steiner and Redish (2014) found that

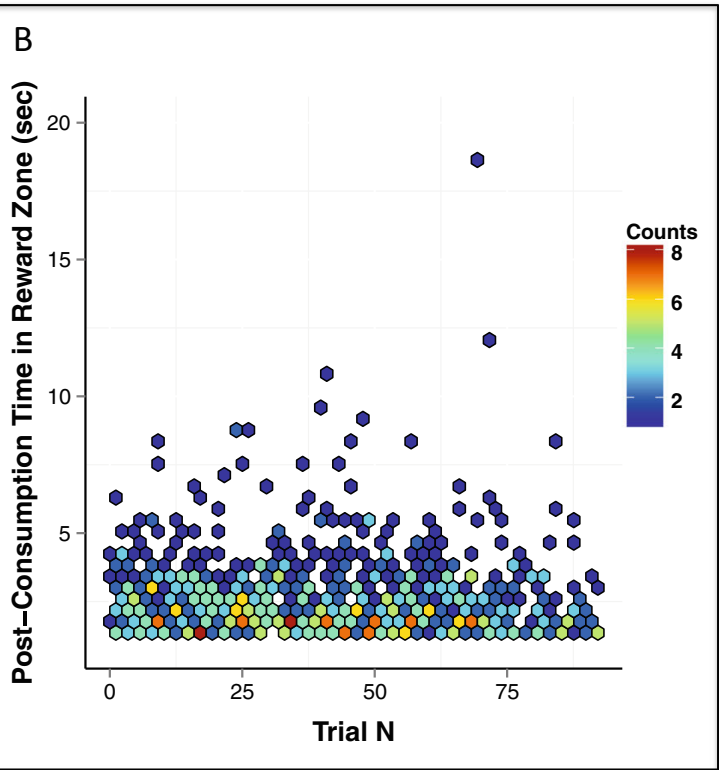

viewing a stimulus (video or photo) but before providing a rating. The counts reflect the number of samples included in a given cell

representations in both orbitofrontal cortex (OFC) and ventral striatum (vStr) reliably tracked choices and preferences (e.g., neuronal signals in these areas differentiated between feeders during reward receipt). Breton et al. (2014) found that compromising OFC with DREADD-driven pyramidal-cell inhibition led to a disruption in flavor preferences, while Schmidt et al. (2014) found that compromising medial prefrontal areas (prelimbic and infralimbic) led to a disruption in hesitation during difficult decisions. Although the homologies between rat and human prefrontal areas remain controversial (Preuss, 1995; Uylings, Groenewegen, \& Kolb, 2003), these findings suggest that it would be extremely interesting to compare human neuroimaging findings and rodent neurophysiological findings on these parallel tasks.

For example, these findings are consistent with human neuroimaging findings, whereby studies have shown that medial OFC activation scales proportional to expected reward value (Rushworth, Kolling, Sallet, \& Mars, 2012), the ventromedial prefrontal cortex (suggested to parallel rodent OFC; Ongür \& Price, 2000; Schoenbaum, Roesch, \& Stalnaker, 2006) reflects rewards and decisions (Balleine \& O'Doherty, 2010; Gläscher, Hampton, \& O’Doherty, 2009; Hampton, Bossaerts, \& O'Doherty, 2006), and the dorsolateral prefrontal cortex (suggested to parallel rodent mPFC; Ongür \& Price, 2000; Seamans, Lapish, \& Durstewitz, 2008) links with deliberative decision processes (Krawczyk, 2002). One might also anticipate cross-species parallels in recruitment of the anterior cingulate cortex (ACC; Kolling, Behrens, Mars, \& Rushworth, 2012). For example, evidence suggests that the ACC may monitor performance, such as the yield of foraging decisions. In particular, the ACC is sensitive to situations where the 
alternative value is deemed greater than the current option, thus leading the subject to skip. Lastly, the anterior insula is an additional target region for tracking reward responsiveness during the Web-Surf Task, as this area is closely linked to the salience network and has been shown to activate more strongly in response to primary than secondary rewards in humans (Sescousse, Caldú, Segura, \& Dreher, 2013).

\section{Conclusions}

Collectively, our findings support the use of the Web-Surf Task as an effective experiential human foraging paradigm. Many decision-making tasks are concerned with modeling the motivation (or aversion) to reward and punishment as a means to characterize impulse-related psychopathology. To effectively model reward requires that a given task capture the natural ethology of a species - a reason that has led many to utilize monetary questionnaire or point-based delaydiscounting paradigms. However, money is only symbolically rewarding to humans, and is not a comparable primary reinforcer as the food rewards used in rodent paradigms. Our results demonstrate that video stimuli provide a compelling counterpart to food that can be easily incorporated into an experimental setup. Moreover, the multi-option design enables researchers to evaluate individual differences in preference. This feature may be valuable for researchers interested in mapping various behavioral parameters with other marks of impulsivity (e.g., self-report, neural activation, etc.). Therefore, this research lays the foundation for a stream of functional translational research that seeks to narrow the gap between pre-clinical and clinical research via parallel tasks.

Acknowledgments This study was supported by grants from the National Institute on Drug Abuse (NIDA) to Samantha Abram (F31DA040335-01), Brandy Schmidt (F32-DA038392-01A1 and R01DA030672S1), and A. David Redish (R01-DA030672). Brandy Schmidt's contributions to this study were also supported by the Society for Neuroscience's (SfN) Neuroscience Scholars Program.

\section{References}

Balleine, B. W., \& O'Doherty, J. P. (2010). Human and rodent homologies in action control: Corticostriatal determinants of goal-directed and habitual action. Neuropsychopharmacology, 35(1), 48-69. doi: 10.1038/npp.2009.131

Belzung, C., \& Lemoine, M. (2011). Criteria of validity for animal models of psychiatric disorders: Focus on anxiety disorders and depression. Biology of Mood \& Anxiety Disorders, 1(1), 9. doi:10. 1186/2045-5380-1-9

Blatter, K., \& Schultz, W. (2006). Rewarding properties of visual stimuli. Experimental Brain Research, 168, 541-546. doi:10.1007/s00221005-0114-y

Bohbot, V. D., Lerch, J., Thorndycraft, B., Iaria, G., \& Zijdenbos, A. P. (2007). Gray matter differences correlate with spontaneous strategies in a human virtual navigation task. The Journal of Neuroscience, 27(38), 10078-10083. doi:10.1523/JNEUROSCI.1763-07.2007

Bradley, D. B., Katherine, D. D., Dylan, S. A., Daphna, S., \& Nathaniel, D. D. (2015). Model-based choices involve prospective neural activity. Nature Neuroscience, 18(5), 767-772.

Breton, Y., Schmidt, B., \& Redish, A. (2014). Orbitofrontal inactivation blunts flavor preferences in the Restaurant Row task. In Society for Neuroscience. Washington, DC.

Demeter, E., Sarter, M., \& Lustig, C. (2008). Rats and Humans Paying Attention. Neuropsychology, 22(6), 787-799. doi:10.1037/ a0013712.Rats

Ding, W., Sun, J., Sun, Y., Zhou, Y., Li, L., Xu, J., \& Du, Y. (2013). Altered Default Network Resting-State Functional Connectivity in Adolescents with Internet Gaming Addiction. PLOS ONE, 8(3). doi: 10.1371/journal.pone.0059902

Feighery, E. C., Schleicher, N. C., Boley Cruz, T., \& Unger, J. B. (2008). An examination of trends in amount and type of cigarette advertising and sales promotions in California stores, 2002-2005. Tobacco Control, 17(2), 93-98. doi:10. $1136 /$ tc. 2007.022046

Gläscher, J., Hampton, A. N., \& O’Doherty, J. P. (2009). Determining a role for ventromedial prefrontal cortex in encoding action-based value signals during reward-related decision making. Cerebral Cortex, 19(2), 483-495. doi:10.1093/cercor/bhn098

Grossman, M., \& Chaloupka, F. J. (1998). The demand for cocaine by young adults: A rational addiction approach. Journal of Health Economics, 17(4), 427-474. doi:10.1016/ S0167-6296(97)00046-5

Hall, Z. J., De Serrano, A. R., Rodd, F. H., \& Tropepe, V. (2014). Casting a wider fish net on animal models in neuropsychiatric research. Progress in Neuro-Psychopharmacology \& Biological Psychiatry, 55, 7-15. doi:10.1016/j.pnpbp.2014.04.003

Hamilton, D. A., Driscoll, I., \& Sutherland, R. J. (2002). Human place learning in a virtual Morris water task: Some important constraints on the flexibility of place navigation. Behavioural Brain Research, 129(1-2), 159-170. doi:10.1016/S01664328(01)00343-6

Hamilton, D. A., Kodituwakku, P., Sutherland, R. J., \& Savage, D. D. (2003). Children with Fetal Alcohol Syndrome are impaired at place learning but not cued-navigation in a virtual Morris water task. Behavioural Brain Research, 143(1), 85-94. doi:10.1016/S01664328(03)00028-7

Hampton, A. N., Bossaerts, P., \& O’Doherty, J. P. (2006). The role of the ventromedial prefrontal cortex in abstract statebased inference during decision making in humans. The Journal of Neuroscience, 26(32), 8360-8367. doi:10.1523/ JNEUROSCI.1010-06.2006

Haxby, J. V., Ungerleider, L. G., Clark, V. P., Schouten, J. L., Hoffman, E. A., \& Martin, A. (1999). The effect of face inversion on activity in human neural systems for face and object perception. Neuron, 22(1), 189-199. doi:10.1016/S0896-6273(00)80690-X

Haxby, J. V., Gobbini, M. I., Furey, M. L., Ishai, A., Schouten, J. L., \& Pietrini, P. (2001). Distributed and Overlapping Representations of Faces and Objects in Ventral Temporal Cortex. Science, 293(5539), 2425-2430. doi:10.1126/science.1063736

Hayden, B. Y., Pearson, J. M., \& Platt, M. L. (2011). Neuronal basis of sequential foraging decisions in a patchy environment. Nature Neuroscience, 14, 933-939. doi:10.1038/nn.2856

Hoffer, L. D., Bobashev, G., \& Morris, R. J. (2009). Researching a local heroin market as a complex adaptive system. American Journal of Community Psychology, 44(3-4), 273-286. doi:10.1007/s10464-0099268-2

Iaria, G., Petrides, M., Dagher, A., Pike, B., \& Bohbot, V. D. (2003). Cognitive strategies dependent on the hippocampus and caudate nucleus in human navigation: Variability and 
change with practice. The Journal of Neuroscience, 23(13), $5945-5952$.

Kalivas, P. W., Peters, J., \& Knackstedt, L. (2006). Animal Models and Brain Circuits in Drug Addiction. Molecular Interventions, 6(6), 339-344.

Kolling, N., Behrens, T. E. J., Mars, R. B., \& Rushworth, M. F. S. (2012). Neural Mechanisms of Foraging. Science, 336(6077), 95-98. doi: $10.1126 /$ science. 1216930

Kool, W., \& Botvinick, M. M. (2014). A labor/leisure tradeoff in cognitive control. Journal of Experimental Psychology. General, 143(1), 131-141.

Krawczyk, D. C. (2002). Contributions of the prefrontal cortex to the neural basis of human decision making. Neuroscience and Biobehavioral Reviews, 26(6), 631-664. doi:10.1016/S01497634(02)00021-0

Krishnan-Sarin, S., Reynolds, B., Duhig, A. M., Smith, A., Liss, T., McFetridge, A., ... Potenza, M. N. (2007). Cessation Program for Adolescent Smokers. Drug and Alcohol Dependence, 88(1), 79-82.

Kuss, D. J., \& Griffiths, M. D. (2012). Internet Gaming Addiction: A Systematic Review of Empirical Research. International Journal of Mental Health and Addiction, 10(2), 278-296. doi:10.1007/s11469011-9318-5

Madden, G. J., \& Bickel, W. K. (Eds.). (2010). Impulsivity: The Behavioral and Neurological Science of Discounting. Washington, DC: American Psychological Association. doi:10.1016/j.biopsych. 2009.08.004.Found

Mazur, J. (1987). An Adjusting Procedure for Studying Delayed Reinforcement. In M. Commons, J. E. Mazur, J. A. Nevin, \& H. Rachlin (Eds.), The effect of delay and intervening events on reinforcement value (pp. 55-73). Hillsdale: Erlbaum. doi:10.1016/j.pbb. 2008.06.017

McClure, S. M., Ericson, K. M., Laibson, D. I., Loewenstein, G., \& Cohen, J. D. (2007). Time Discounting for Primary Rewards. The Journal of Neuroscience, 27(21), 5796-5804. doi:10.1523/ JNEUROSCI.4246-06.2007

Moffat, S. D., Hampson, E., \& Hatzipantelis, M. (1998). Navigation in a "Virtual" Maze: Sex Differences and Correlation With Psychometric Measures of Spatial Ability in Humans. Evolution and Human Behavior, 19(2), 73-87. doi:10.1016/S1090-5138(97) 00104-9

Montague, P. R., Dolan, R. J., Friston, K. J., \& Dayan, P. (2012). Computational psychiatry. Trends in Cognitive Sciences, 16(1), 72-80. doi:10.1016/j.tics.2011.11.018

O’Brien, C. P., \& Gardner, E. L. (2005). Critical assessment of how to study addiction and its treatment: Human and non-human animal models. Pharmacology \& Therapeutics, 108(1), 18-58. doi:10. 1016/j.pharmthera.2005.06.018

Ongür, D., \& Price, J. L. (2000). The organization of networks within the orbital and medial prefrontal cortex of rats, monkeys and humans. Cerebral Cortex, 10(3), 206-219. doi:10.1093/cercor/10.3.206

Papale, A. E., Stott, J. J., Powell, N. J., Regier, P. S., \& Redish, A. D. (2012). Interactions between deliberation and delay-discounting in rats. Cognitive, Affective, \& Behavioral Neuroscience, 12(3), 513526. doi:10.3758/s13415-012-0097-7

Peelen, M. V., \& Downing, P. E. (2007). The neural basis of visual body perception. Nature Reviews Neuroscience, 8, 636-648. doi:10.1038/ nrn2195

Peirce, J. W. (2009). Generating Stimuli for Neuroscience Using PsychoPy. Frontiers in Neuroinformatics, 2(10). doi:10.3389/neuro.11.010.2008

Pirolli, P. (2005). Rational Analyses of Information Foraging on the Web. Cognitive Science, 29, 343-373.

Pirolli, P., \& Card, S. (1999). Information foraging. Psychological Review, 106(4), 643-675. doi:10.1037/0033-295X.106.4.643

Potenza, M. N. (2009). The Importance of Animal Models of Decision Making, Gambling, and Related Behaviors: Implications for Translational Research in Addiction. Neuropsychopharmacology, 34(13), 2623-2624. doi:10.1038/npp.2009.152
Preuss, T. M. (1995). Do Rats Have Prefrontal Cortex? The RoseWoolsey-Akert Program Reconsidered. Journal of Cognitive Neuroscience, 7(1), 1-24. doi:10.1162/jocn.1995.7.1.1

Rangel, A., Camerer, C., \& Montague, P. R. (2008). A framework for studying the neurobiology of value-based decision making. Nature Reviews. Neuroscience, 9(7), 545-556. doi:10. $1038 /$ nrn2357

Redish, A. D. (2013). The Mind Within the Brain: How We Make Decisions and How Those Decisions Go Wrong. (O. U. Press, Ed.) (1st ed., p. 392). New York.

Redish, A. D., Jensen, S., \& Johnson, A. (2008). A unified framework for addiction: Vulnerabilities in the decision process. The Behavioral and Brain Sciences, 31(4), 415-437. doi:10.1017/ S0140525X08004986. discussion 437-487.

Reynolds, B. (2006a). A review of delay-discounting research with humans: Relations to drug use and gambling. Behavioural Pharmacology, 17(8), 651-667. doi:10.1097/FBP. 0b013e3280115f99

Reynolds, B. (2006b). The Experiential Discounting Task is sensitive to cigarette- smoking status and correlates with a measure of delay discounting. Behavioural Pharmacology, 17(2), 133-142.

Reynolds, B., \& Schiffbauer, R. (2004). Measuring state changes in human delay discounting: An experiential discounting task. Behavioural Processes, 67(3), 343-356. doi:10.1016/j.beproc.2004.06.003

Rushworth, M. F., Kolling, N., Sallet, J., \& Mars, R. B. (2012). Valuation and decision-making in frontal cortex: One or many serial or parallel systems? Current Opinion in Neurobiology, 22(6), 946-955. doi:10. 1016/j.conb.2012.04.011

Schmidt, B., Breton, Y., \& Redish, A. (2014). Silencing the rat medial prefrontal cortex decreases hesitation and impairs vicarious trial and error (VTE) behavior on the Restaurant Row task. In Society for Neuroscience. Washington, DC.

Schoenbaum, G., Roesch, M. R., \& Stalnaker, T. A. (2006). Orbitofrontal cortex, decision-making and drug addiction. Trends in Neurosciences, 29(2), 116-124. doi:10.1016/j.tins.2005.12.006

Schüll, N. (2012). Addiction by design: Machine gambling in Las Vegas. The Annals of the American Academy of Political and Social Science, 597, 65-81. doi:10.2307/j.ctt12f4d0

Seamans, J. K., Lapish, C. C., \& Durstewitz, D. (2008). Comparing the prefrontal cortex of rats and primates: Insights from electrophysiology. Neurotoxicity Research, 14(2-3), 249-262. doi:10.1007/ BF03033814

Sescousse, G., Caldú, X., Segura, B., \& Dreher, J. C. (2013). Processing of primary and secondary rewards: A quantitative meta-analysis and review of human functional neuroimaging studies. Neuroscience and Biobehavioral Reviews, 37(4), 681-696. doi:10.1016/j. neubiorev.2013.02.002

Shenhav, A., Straccia, M. A., Cohen, J. D., \& Botvinick, M. M. (2014). Anterior cingulate engagement in a foraging context reflects choice difficulty, not foraging value. Nature Neuroscience, 17, 1249-1254. doi:10.1038/nn.3771

Steiner, A. P., \& Redish, A. D. (2014). Behavioral and neurophysiological correlates of regret in rat decision-making on a neuroeconomic task. Nature Neuroscience, 17(7), 9951002. doi: $10.1038 / \mathrm{nn} .3740$

Stephens, D. (2008). Decision ecology: Foraging and the ecology of animal decision making. Cognitive, Affective, \& Behavioral Neuroscience, 8(4), 475-484. doi:10.3758/CABN.8.4.475

Uylings, H. B. M., Groenewegen, H. J., \& Kolb, B. (2003). Do rats have a prefrontal cortex? Behavioural Brain Research, 146(1-2), 3-17. doi: 10.1016/j.bbr.2003.09.028

Voon, V., Reynolds, B., Brezing, C., Gallea, C., Skaljic, M., Ekanayake, V., ... Hallett, M. (2010). Impulsive choice and response in dopamine agonist-related impulse control behaviors. Psychopharmacology, 207(4), 645-659. doi:10.1007/s00213-009-1697-y 
Walther, D. B., Caddigan, E., Fei-Fei, L., \& Beck, D. M. (2009). Natural scene categories revealed in distributed patterns of activity in the human brain. The Journal of Neuroscience: The Official Journal of the Society for Neuroscience, 29(34), 10573-10581. doi:10. 1523/JNEUROSCI.0559-09.2009

Weinstein, A., \& Lejoyeux, M. (2015). New developments on the neurobiological and pharmaco-genetic mechanisms underlying internet and videogame addiction. American Journal on Addictions, 24(2), 117-125. doi:10.1111/j.1521-0391.2013.12110.x

Wikenheiser, A. M., Stephens, D. W., \& Redish, A. D. (2013). Subjective costs drive overly patient foraging strategies in rats on an intertemporal foraging task. Proceedings of the National Academy of Sciences, 110(20), 8308-8313. doi:10.1073/ pnas. 1220738110 